\title{
A173 LOSS OF THE WNT INHIBITOR SCLEROSTIN PROMOTES PANNUS FORMATION AND ACCELERATES JOINT DESTRUCTION IN THE HTNFTG MOUSE MODEL OF RHEUMATOID ARTHRITIS
}

Corinna Wehmeyer, ${ }^{1}$ Athanasios Stratis, ${ }^{1}$ Ina Kramer, ${ }^{2}$ Michaela Kneissel, ${ }^{2}$ Thomas Pap, ${ }^{1}$ Berno Dankbar ${ }^{1}$ IInstitute of Experimental Musculoskeletal Medicine - IEMM, University Hospital Muenster, Germany; ${ }^{2}$ Novartis Institutes for BioMedical Research, Basel, Switzerland

\subsection{6/ard.2010.149013.16}

Background In rheumatoid arthritis (RA), progressive joint destruction is a hallmark of disease and results from both increased bone resorption and the lack of repair mechanisms. tumour necrosis factor $\alpha$ (TNF $\alpha$ ) contributes to both aspects of pathologic joint remodelling by increasing the number of bone-resorbing osteoclasts and decreasing the number of bone-forming osteoblasts. Sclerostin is a potent inhibitor of osteoblast development by antagonising the Wnt/ $\beta$-catenin signalling pathway. Based on recent data that have shown increased expression of sclerostin under inflammatory conditions, the authors studied its expression in human RA and in human TNF transgenic (hTNFtg) mice, which develop a RA-like destructive arthritis. Moreover, the authors analysed the effects of sclerostin deficiency on the development and severity of the arthritis in these mice.

Methods Expression of sclerostin was determined by immunohistochemistry, western blot and reverse transcriptase PCR. To assess the functional role of sclerostin in vivo, sclerostin knockout $\left(S_{S S T^{-/}}\right)$mice were crossed with hTNFtg mice.

In addition to determining the clinical severity of disease in SOST S- $^{-/}$hTNFtg and hTNFtg mice, histological changes including bone erosion, cartilage destruction and inflammation were evaluated by histomorphometric analyses. The number of osteoclasts was quantified using tartrate-resistant acid phosphatase staining. Immunohistochemistry was performed to analyse the expression of molecules of the Wnt pathway.

Results Immunohistochemistry and western blot analyses revealed a strong overexpression of sclerostin in synovial tissue of RA compared to osteoarthritis patients. Likewise, ankle joints of hTNFtg mice showed high levels of sclerostin, especially in the infiltrating pannus, whereas only negligible staining was observed in wild-type animals. In vitro, expression of sclerostin was only found in osteoblasts and osteoclasts, but could be induced in RA synovial fibroblasts by TNF $\alpha$. Surprisingly, the lack of sclerostin not only increased the clinical severity of arthritis in hTNFtg mice but most dramatically accelerated joint damage in this mouse model of RA. SOST ${ }^{-1-}$ hTNFtg mice displayed significant higher bone erosion, synovial hyperplasia and osteoclast numbers compared to hTNFtg mice. Moreover, immunohistochemistry revealed higher levels of dickkopf 1 (DKK-1) and Wnt-5a in joints of SOST-deficient hTNFtg mice.

Conclusions The authors hypothesise that under inflammatory conditions, higher levels of DKK-1 and Wnt-5a in joints of sclerostin-deficient arthritic mice counteract the beneficial effect of sclerostin deficiency by increasing osteoclast development through enhanced blockade of the Wnt-3a pathway by DKK-1 as well as by promoting synovial hyperplasia through Wnt-5a-mediated synoviocyte activation. These results may have an impact on the use of sclerostin inhibitors in inflammatory joint diseases. 